\title{
Determination of winding diameter based on bending strain analysis for REBCO coated conductor tapes
}

\author{
M. B. de Leon, M. J. Dedicatoria and H. S. Shin* \\ Department of Mechanical Engineering, Andong National University, Andong, Kyungbuk 760-749, Korea
}

Received 22 March 2012; accepted 19 April 2012

\begin{abstract}
In order to recognize the allowable bending diameter in coils, the strain as function of diameters is evaluated. The irreversible strain limits of $I_{c}$ in the easy and hard bending modes were measured. Strains were calculated at the coating film in the easy bending and at outer edge or inner edge in the hard bending of the $\mathrm{CC}$ tape, respectively. The tape geometry subjected to bending procedures is considered from the current industrial spool winding operation. Through the linear superposition of strain induced in different bending modes regarding the expressions, the appropriate design for critical bending diameter is suggested. Results proved that the existence of buckling resulting from bending in hard direction when applied strain exceeded $0.6 \%$ is possible. The depicted results showed that the strain limit as a viable parameter should be considered for future purposes.
\end{abstract}

\section{INTRODUCTION}

Remarkable progress in making long length coated conductor (CC) tapes with high critical current, $I_{c}$ has already been achieved. Recently, great concerns in manufacturing include increasing the capacity to reduce cost, and improving process consistency to improve product homogeneity and predictability of performance for risk reduction [1]

Since REBCO CC tapes are known as a robust product with good electromechanical properties, it is expected to be used in power dense coils such as motors, generators and magnets. However, the $I_{\mathrm{c}}$ of REBCO CC tapes are basically strain dependent, in the sense that the strain induced should not exceed the irreversible strain limit, $\varepsilon_{\text {irr }}$ [2]. The measurement of $I_{c}$ degradation with strain is important to determine the bending diameter limit for the size of spools or the minimum bending diameter of the pancake coil for winding fabrication. In addition, the bending strain limits should be considered when winding into coils.

In this study, the well-known double pancake winding method was investigated. This method was suitable for winding thin and rectangular-shaped tapes that features a typical YBCO CC. Using this fabrication method, the tape is wound in radial disks or pancakes, in pairs connected at the inner winding diameter by a joint or, alternatively, a continuous transition from one disk to the other [2]. The latter has the advantage to be wound at particular small diameters and the possibility to avoid any joints within the

* Corresponding author: hsshin@andong.ac.kr

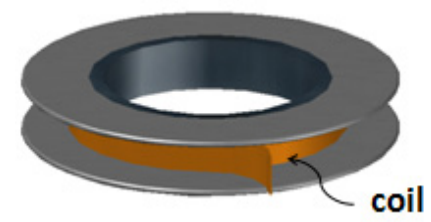

(a)

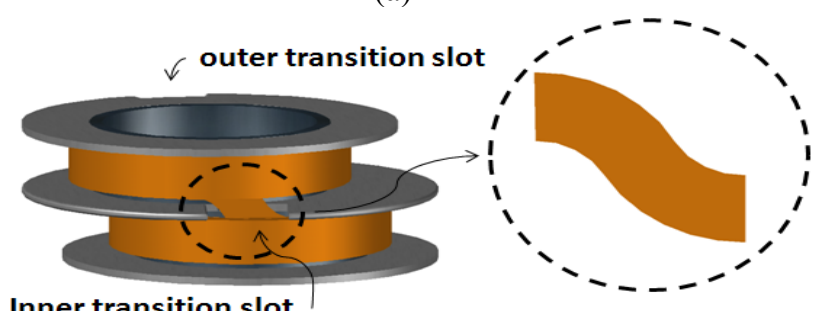

(b)

Fig. 1. a) Single pancake unit; b) a double pancake unit with slot for transitions and possible joint region.

pancake. Fig. 1 shows the assembled mechanical structure of the (a) single pancake unit and (b) double-pancake unit $[3]$.

The objective of this study is to determine the critical winding diameter in pancake coil winding based on the bending strain analysis in case that REBCO CC tape is adopted. Recently, other research group already investigated the double pancake coils through critical current and over-current tests [4]. A YBCO CC tape was selected and $I_{c}$ was measured as a function of bending strain $\left(\varepsilon_{\mathrm{b}}\right)$ during both easy and hard bending tests. $I_{c}$ is normalized by the critical current measured at the unbend condition, $I_{c 0}$ and is expressed as $I_{c} / I_{c 0}$. Consequently, the total bending strain $\left(\varepsilon_{\text {total,YBCO }}\right)$ generated for both bending modes was evaluated as a function of winding diameter for the case of double pancake coil.

\section{EXPERIMENTAL PROCEDURES}

\subsection{Samples}

A commercially available $\mathrm{YBa}_{2} \mathrm{Cu}_{3} \mathrm{O}_{7-x} \mathrm{CC}$ tapes were supplied for the test and the properties are tabulated in Table I. A $80 \mu \mathrm{m}$ thick Ni-5 at \% W alloy substrate was prepared by the rolling assisted bi-axially textured substrate (RABITS) technique. The YBCO layer with about $1 \mu \mathrm{m}$ thick was deposited using metal organic deposition (MOD). Copper stabilizer of about $40 \mu \mathrm{m}$ on 
TABLE I

SPECIFICATIONS OF YBCO COATED CONDUCTOR TAPE SAMPLE.

\begin{tabular}{lc}
\hline & $\mathrm{RABiTS} / \mathrm{MOD}$ \\
\hline Structure & $\mathrm{Ag} / \mathrm{YBCO} / \mathrm{Ce}_{2} / \mathrm{YSZ} / \mathrm{Y}_{2} \mathrm{O}_{3}$ \\
YBCO film thickness & about $1 \mu \mathrm{m}$ \\
$\mathrm{I}_{\mathrm{c}}$ & $86 \mathrm{~A}$ \\
Dimension, T x W & $0.19 \times 4.3 \mathrm{~mm}$ \\
Substrate & $\mathrm{Ni}-5 \mathrm{at} . \% \mathrm{~W}$ \\
Substrate thickness & $80 \mu \mathrm{m}$ \\
Stabilizer & Hardened copper \\
Stabilizing technique & Lamination (solder) \\
Stabilizer thickness & $40 \mu \mathrm{m}$ \\
Manufacturer & AMSC \\
\hline
\end{tabular}

both sides protects the conductor and produces rounded edges that are beneficial for high-voltage applications. Sample edges are hermetically sealed with solders on both sides. The samples are specified as MOD-RABITS.

It is necessary to know the location of the neutral axis on the cross-section in order to accurately measure the bending strain induced in the coating film layer. Since the YBCO layer and buffer layers are relatively thin, it can be regarded as that the neutral axis for bending may be taken to be at the center of the substrate [2].

\subsection{Measurement techniques}

The bending strain limit of $I_{\mathrm{c}}$ in the YBCO CC tape was measured by using a small scale Goldacker test rig for the easy bending test and GFRP mandrels having different radii for the hard bending test. Using the continuous easy bending test rig, both tensile and compressive bending strain can be applied to the sample depending on whether the YBCO layer is in the inside or outside layer of the bent specimen, namely, below or above the neutral axis.

With the bending analysis in thin beams, the winding to an individual pancake and the inner diameter transition between pancakes will experience the combination of bending in easy (thickness wise) and hard (width wise) mode. In addition, the bending in each mode will be a component for the calculation of strain and further discussion is in section 3 .

\subsubsection{Easy bending mode}

Fig. 2(a) shows the small scale Goldacker test rig used to measure the bending strain dependence of the critical current, $I_{c}\left(\varepsilon_{\mathrm{b}}\right)$, in easy bending mode. The $I_{c}$ was measured by the four probe method and defined by a criterion of 1 $\mu \mathrm{V} / \mathrm{cm}$. The CC sample is clamped by both side grips and continuous bending application is possible to give uniform deformation in the shape of a circular arc. In this case, the total sample length, voltage tap separation, location of the turn axis, and the gauge length subjected to easy bending deformation were $60,10,11.8$ and $18.5 \mathrm{~mm}$ respectively. The detail of Goldacker bending test procedure is described in our previous report [5].

\subsubsection{Hard bending mode}

Considering the bending of the $\mathrm{CC}$ tape in the case of

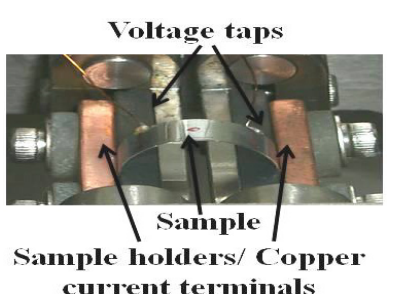

(a)

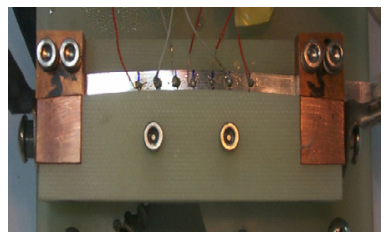

(b)
Fig. 2. a) Goldacker test rig (easy bending) and b) bending mandrel (hard bending) for measuring the bending strain dependence of $I_{c}$.

double pancake winding subjected through a continuous transition at the inner winding diameter, then, the measurement of $I_{c}$ degradation in hard bending mode is necessary. The bending strain was applied to the CC tapes at room temperature (RT) across the width. Sample was mechanically fixed to the copper terminals in a bent position on a curved GFRP sample holder. Then, the $I_{c}$ was measured after cooling down to $77 \mathrm{~K}$. Fig. 2 (b) shows the appearance of sample mounted to GFRP holder in hard bending mode. Multiple voltage taps (A to $G$ ) were adopted to check the homogeneity of $I_{\mathrm{c}}$ degradation within the whole AG section. The details of testing procedure are described in our previous report $[5,6]$. $I_{\mathrm{c}}$ was measured at specific bending strains and also after releasing the bending strain to measure its recoverability and to determine the irreversible strain limit $\left(\varepsilon_{\text {irr }}\right)$.

\section{BENDING STRAIN CALCULATION}

The bending geometry of $\mathrm{CC}$ tape and the strain induced at the coating film in easy bending mode is observed and evaluated as illustrated in Fig. 3(a). Applying the standard bending strain expression, the bending strain in easy bending mode can be expressed as

$$
\varepsilon_{\mathrm{b}}=\left(y / r_{\mathrm{t}}\right)
$$

where $\varepsilon_{\mathrm{b}}$ is the bending strain, $y$ is the distance to the YBCO coating film layer from the neutral axis and $r_{\mathrm{t}}$ is the radius of curvature to the conductor neutral axis which is equal to the bending radius plus a half of the conductor thickness. Then the corresponding strain at the location of YBCO coating film is given by

$$
\varepsilon_{\mathrm{eb}, \mathrm{YBCO}}= \pm t_{\mathrm{s}} /(2 r+t)
$$

where $t$ and $t_{\mathrm{s}}$ represent the thickness of CC sample and substrate layer, respectively and $r$ is the bending radius with respect to sample's curvature. The bending strain is tensile $(+)$ or compressive (-) depending on the location of the YBCO layer in the CC tape configuration.

The strain in the easy bending mode for the YBCO CC tapes is given in (1). This technique allowed us to determine the bending diameter limit of YBCO CC tape which corresponds to the irreversible strain limit $\left(\varepsilon_{\text {irr }}\right)$. However, in the actual design and process of double pancake coil with inner transition, this bending strain limit only accounts for the single coil. The strain induced due to 


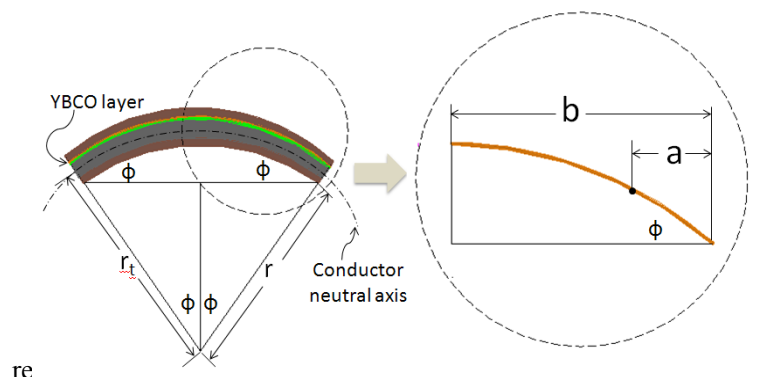

(a)

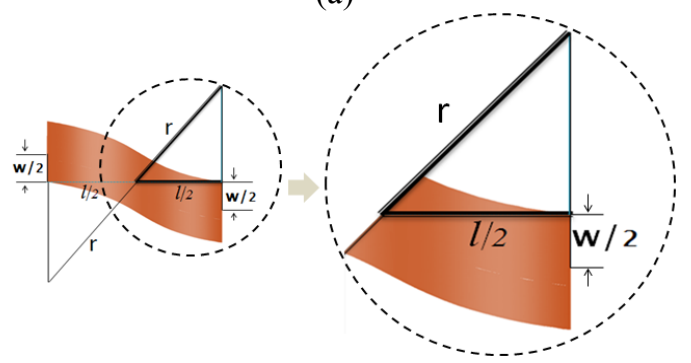

(b)

Fig. 3. a) Geometry of YBCO CC sample in easy bending; b) hard bending geometry.

hard bending at the continuous transition should also be considered. For hard bending in the width direction, as described in Fig. 3 (b), using the analysis of Markiewicz and Swenson [2], when the transition occupies a complete turn, the maximum strain at the tape is given by the expression

$$
\varepsilon_{\mathrm{hb}, \text { YBCO }}= \pm\left(2 \mathrm{w}^{2} / l^{2}\right)\left\{1 /\left[1+(\mathrm{w} / l)^{2}\right]\right\}
$$

where $w$ is the tape width and $l$ is the circumferential length. The (+) and (-) signs are the bend strains on the outside and the inside curvature of the bend, respectively. Furthermore, when a full circumference is used for the transition, the minimum strain can be calculated.

Then, the total strain induced in coiling the YBCO CC tape at the transition can be expressed as the summation of eq's (2) and (3).

$$
\varepsilon_{\text {total, } \mathrm{YBCO}}=\varepsilon_{\mathrm{eb}, \mathrm{YBCO}}+\varepsilon_{\mathrm{hb}, \mathrm{YBCO}}
$$

\section{RESULT S AND DISCUSSION}

The understanding of $I_{\mathrm{c}}$ degradation with strain is important in practical device applications. Previous studies showed that the irreversible $I_{\mathrm{c}}$ degradation has been attributed to cracking on the YBCO film layer [5-7]. In this study, $I_{c}$ was investigated under tensile and compressive bending. The coated conductor was bent successively up to the smallest possible diameter of the bending test rig and then back to the straight position to measure whether the $I_{\mathrm{c}}$ recovers to $99 \% I_{\mathrm{c} 0}$.

Using continuous bending, the tolerable strain defined at $95 \% I_{c}$ retention was in the range of $0.35 \sim 0.45 \%$ and it is confirmed to be reversible both in tension and compression, as shown in Fig 4 (a). Reversibility test was done when $I_{\mathrm{c}}$ degraded by $5 \%$. $I_{c}$ fully recovers when straightened which clearly means that no permanent damage has been brought on YBCO coating film layer like

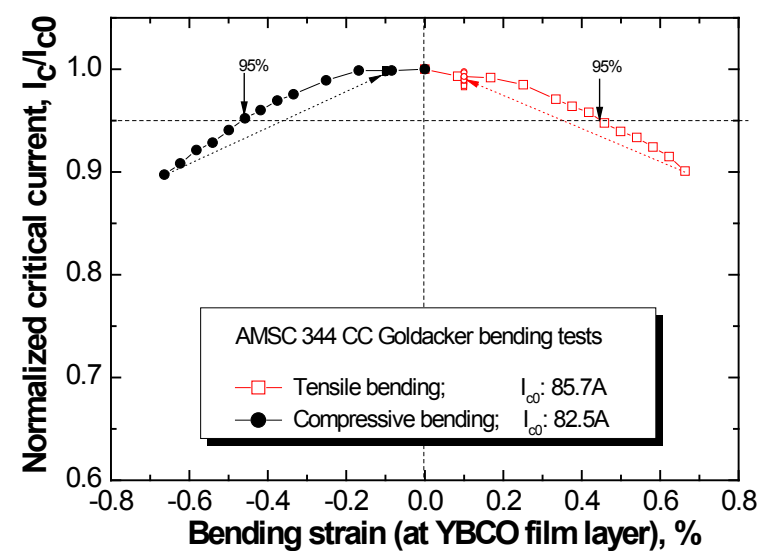

(a)

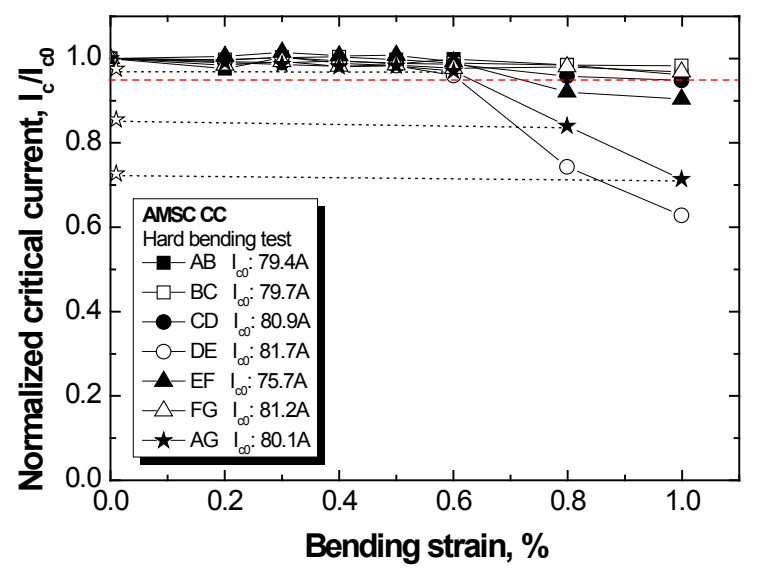

(b)

Fig. 4. a) $I_{c} / I_{c 0}-\varepsilon_{\mathrm{b}}$ relationship of the Cu-laminated YBCO $\mathrm{CC}$ tape. b) $I_{\mathrm{c}} / I_{\mathrm{c} 0}-\varepsilon_{\mathrm{b}}$ relationships in $\mathrm{YBCO} \mathrm{CC}$ tapes obtained during hard bending tests.

cracks as what has been described in our previous report [6]. The reversibility of $I_{\mathrm{c}}$ with bending strain was retained up to $0.65 \%$ which is the bending test rig limit, but cracking did not occur on the YBCO film layer. Based on the result, although the $I_{c}$ is reversible up to the $0.65 \%$ bending strain at the YBCO film layer, it is also important to note that the $I_{\mathrm{c}}$ had already decreased by $10 \%$. Current capacity of the coil is a primary parameter for the designer for commercial viability and it should be considered crucial for the coil winding criteria.

On the other hand, in the case of hard bending test, homogeneity in the tape was observed up to bending strain $\left(\varepsilon_{\mathrm{hb}}\right)$ of $1 \%$ along the longitudinal direction in YBCO CC tape and the details were previously reported on [8]. The results showed no degradation and little variation in critical current $\left(I_{c}\right)$ up to $0.6 \%$ bending strain $\left(\varepsilon_{\mathrm{hb}}\right)$ as can be observed in Fig. 4(b). However, $I_{c}$ started to degrade depending on the location of sub-sections when the bending strain $\left(\varepsilon_{\mathrm{hb}}\right)$ exceeded $0.6 \%$. Regions DE and EF, where local buckling occurred, showed a significant $I_{c}$ degradation and failed to recover when bending strain was released. This buckling might be related to torsion or turnaround that resulted to significant damage to $\mathrm{YBCO}$ 


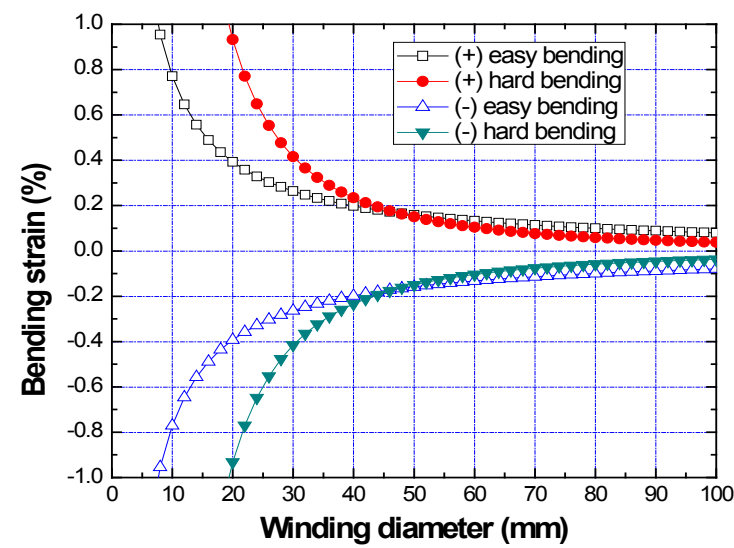

Fig. 5. Relationship of winding diameter and its corresponding bending strain at the YBCO film in easy and hard bending.

TABLE II

TABUlated Results For EASY AND HARD BENDING.

\begin{tabular}{ccc}
\hline $\begin{array}{c}\text { Winding } \\
\text { diameter } \\
(\mathrm{mm})\end{array}$ & $\begin{array}{c}\text { Bending strain, \% } \\
\left(\varepsilon_{\mathrm{eb}}\right) \text { easy mode }\end{array}$ & $\begin{array}{c}\text { Bending strain, \% } \\
\left(\varepsilon_{\mathrm{hb}}\right) \text { hard mode }\end{array}$ \\
\hline 12 & 0.65 & 2.57 \\
16 & 0.61 & 1.46 \\
18 & 0.49 & 1.89 \\
24 & 0.33 & 0.65 \\
25 & 0.32 & 0.60 \\
26 & 0.31 & 0.55 \\
30 & 0.26 & 0.42 \\
32 & 0.25 & 0.37 \\
33 & 0.24 & 0.34 \\
34 & 0.23 & 0.32 \\
\hline
\end{tabular}

film layer. Based on these results, as shown in Fig. 5 and Table II, the $0.65 \%$ bending strain at the YBCO coating film in easy bending is considered to be viable for winding single pancake coil up to nearly $12 \mathrm{~mm}$ diameter (considering only the reversible strain limit). But, for the $95 \% I_{c}$ retention strain limit, the $0.35 \%$ to $0.45 \%$ bending strain $\left(\varepsilon_{\mathrm{b}}\right)$ (corresponding to a bending diameter limit of $17.6 \mathrm{~mm}$ ) are considered to be more practical in applications. Moreover, the inner transition diameter of nearly $25 \mathrm{~mm}$ was enough for the bending strain $\left(\varepsilon_{\mathrm{b}}\right)$ to be reversible in hard bending test only. But considering at the continuous inner transition that the $\mathrm{CC}$ tape is experiencing both easy and hard bending modes, the total strain experienced in the coating film should be the superposition of $\varepsilon_{\mathrm{eb}}$ and $\varepsilon_{\mathrm{hb}}\left(\varepsilon_{\mathrm{total}}=\varepsilon_{\mathrm{eb}}+\varepsilon_{\mathrm{hb}}\right)$. Since the $I_{\mathrm{c}}$ is reversible under the condition $\varepsilon_{\mathrm{eb}} \leq 0.65 \%$ and $\varepsilon_{\mathrm{hb}} \leq 0.60 \%$, therefore, the allowable total strain $\left(\varepsilon_{\mathrm{t} \text {, allow }}\right)$ should be $<\varepsilon_{\mathrm{hb} . \text { irr }}=0.60 \%$. Then, by the linear superposition, the minimum winding diameter for pancake coil was determined to be $33 \mathrm{~mm}$. This corresponds to $0.58 \%$ total bending strain near the edge part of YBCO film layer. This criterion has been made since we consider that the location of the critical strain will be positioned at the transition. From the analysis of the bending strain in both easy and hard bending modes, the determination of the winding diameter of the coil can be made.

\section{CONCLUSION}

The bending strain in YBCO CC tape in both modes was evaluated by using small scale Goldacker test rig and bending mandrels having different radii, respectively. During continuous easy bending test, the results showed that no permanent damage occurred. The irreversible strain limit during hard bending test was smaller as compared with the case of easy bending test. For the combined strains in easy and hard bending, the allowable total strain $\left(\varepsilon_{\mathrm{t} \text {, allow }}\right)$ should be less than the hard bending irreversibility strain $\left(\varepsilon_{\mathrm{hb} . i r r}=0.60 \%\right)$, since it has the lowest irreversibility strain value. Allowable winding diameter was determined from the critical strain limit at the continuous inner transition. By linear superposition in equation (4), minimum winding diameter for pancake coil was determined to be $33 \mathrm{~mm}$. This corresponds to $0.58 \%$ total bending strain near the edge part of YBCO film layer. Aside from the strain during coiling, it is also necessary to allocate additional strain that will be induced when the coil is energized due to hoop stress and all will comprise the total operating strains.

\section{ACKNOWLEDGMENT}

This work was supported by the National Research Foundation of Korea (NRF) funded by the Korean Government (MEST) (2011-0015369).

\section{REFERENCES}

[1] A. Mbaruku, "Electromechanical and fatigue properties of as-manufactured and quench damaged YBCO coated conductor," PhD Thesis, Florida State Univ., 2006.

[2] W. D. Markiewicz, C. A. Swenson, "Winding strain analysis for YBCO coated conductors." Supercond. Sci. Technol, vol. 23, 045017, March 2010.

[3] A. V. Zlobin et al., "Modeling the high-field section of a Muon helical cooling channel," Proceedings of IPAC'10, Kyoto, Japan, pp. 391-393, 2010.

[4] H. C. Jo et al., "Characteristic comparison of various winding methods of HTS magnets", IEEE Trans. Appl. Supercond., 2012, submitted for publication.

[5] H. S. Shin, K. Katagiri, "Critical current degradation behavior in Bi-2223 superconducting tapes under bending and torsion strains". Supercond. Sci. Technol., vol. 16, pp. 1012-1018, 2003.

[6] H. S. Shin, M. J. Dedicatoria, N. J. Lee, S. S. Oh, "Effect of bending test procedure on the degradation behavior of critical current in ReBCO coated conductor tapes," KIASC Journal, vol. 11 , no. 4, pp. 12-15, 2009

[7] H. S. Shin, J. R. C. Dizon, R. Bonifacio, J. S. Park, "Influence of bending strain on $\mathrm{I}_{\mathrm{c}}$ degradation behavior in $\mathrm{YBCO}$ coated conductor tapes processed using RABiTs /MOD," KIASC Journal, vol. 9, n0. 2, pp. 11-14, 2007.

[8] H. S. Shin, M. J. Dedicatoria, J. R. C. Dizon, H. S. Ha, S. S. Oh, "Bending strain characteristics of critical current in REBCO CC tapes in different modes," Physica C, vol. 469, pp. 1467-1471, 2009.

[9] D. C. van der Laan, J. W. Ekin, C. C. Clickner and T. C. Stauffer, "Delamination strength of YBCO coated conductors under transverse tensile stress," Supercond. Sci. Technol., vol. 20, pp. 765-770, 2007. 